\title{
Drug interaction of ningetinib and gefitinib involving CYP1A1 and efflux transporters in non-small cell lung cancer patients
}

\author{
Lu Liu ${ }^{1}$, Qian $\mathrm{Wang}^{2}$, Cen $\mathrm{Xie}^{3}$, Ning Xi ${ }^{2}$, Zitao $\mathrm{Guo}^{3}$, Ming $\mathrm{Li}^{2}$, Xiangyu $\mathrm{Hou}^{3}$, Ningjie \\ $\mathrm{Xie}^{3}$, Jing $\mathrm{Li}^{2}$, and Xiaoyan $\mathrm{Chen}^{3}$ \\ ${ }^{1}$ Shanghai Institute of Materia Medica Chinese Academy of Sciences \\ ${ }^{2}$ Sunshine Lake Pharma Co Ltd \\ ${ }^{3}$ Shanghai Institute of Materia Medica, Chinese Academy of Sciences
}

May 5, 2020

\begin{abstract}
Background and Purpose: Ningetinib is a tyrosine kinase inhibitor for the treatment of non-small cell lung cancer (NSCLC). When co-administered with gefitinib, the high plasma exposure of $\mathrm{N}$-demethylated metabolite M1 was reduced by more than $80 \%$, whereas it is surprising that of ningetinib was not clearly affected. The present study aims to investigate the drug interaction mechanism of ningetinib and gefitinib. Experimental Approach: NSCLC patients were recruited. Metabolic and transport mechanisms were investigated using in vitro models. Deuterated M1 (D6-M1) and mice were used to study the pharmacokinetic of M1. Key Results: In vitro experiments indicated that CYP1A1 was primarily responsible for M1 formation. Gefitinib was demonstrated to a strong inhibitor of CYP1A1 with Ki value of $0.095 \mu \mathrm{M}$. Co-administration of ningetinib increased blood exposure of intravenously administered D6-M1 by $75 \%$ in mice. M1 was identified as the substrate of the efflux transporters P-gp, BCRP and MRP2, while ningetinib was an inhibitor of these efflux transporters. Consequently, the high plasma exposure of M1 in patients was attributed to its low tissue affinity and the inhibitory effect of the parent drug on M1 canalicular efflux. Conclusion and Implications: When co-administered, gefitinib inhibited the formation of M1 and reduced its plasma exposure, but due to the low metabolic yield of M1 in vivo, the pharmacokinetics of the parent drug ningetinib was not influenced. Inhibition of the CYP1A1 may increase the target tissue concentration of ningetinib. The long-term safety profile and efficacy of ningetinib combined with gefitinib should be concerned in NSCLC patients.
\end{abstract}

\section{What is already known about this subject:}

To improve the efficacy, a combination drug therapy with ningetinib and gefitinib was clinically proposed for the NSCLC treatment.

- $N$-demethyl ningetinib (M1) was identified as the primary circulating metabolite in NSCLC patients whose plasma exposure was about 1.7 -fold that of the parent drug.

- When co-administered with gefitinib, the plasma exposure of M1 was reduced by more than $80 \%$. Contrary to expectations, pharmacokinetics of ningetinib was not clearly affected.

\section{What this study adds:}

- The low tissue affinity and the inhibitory effect of the parent drug on M1 canalicular efflux resulted in the high plasma exposure of M1 in patients

- When co-administrated, the formation of M1 was inhibited by gefitinib, which was firstly demonstrated to a strong inhibitor of CYP1A1.

- Consideration for metabolite kinetics in DDI studies appears to be helpful in elucidating the DDI mechanism. 


\section{Clinical significance:}

- Gefitinib's strong inhibitory effect on CYP1A1 may provide possibilities for its application in tumour prevention and reversal of terminal tumour resistance.

- The long-term safety profile and efficacy of ningetinib combined with gefitinib should be concerned in NSCLC patients.

\section{Introduction}

Non-small cell lung cancer (NSCLC) accounts for approximately $80 \%$ of all cases of lung cancer, and the majority of NSCLC patients present with symptoms in a late advanced stage (Malapelle, Muscarella, Pisapia, \& Rossi, 2020). Currently treatment strategies for NSCLC have evolved to emphasize molecular targeted therapy based on the genomic classification of patients. And the therapeutic targets for NSCLC include epidermal growth factor receptor (EGFR), mechanistic target of rapamycin (mTOR), epidermal growth factor receptor 2 (ErbB2), phosphatidylinositol 3-kinase (PI3Ks), kirsten human rat sarcoma protein (KRAS), vascular epidermal growth factor receptor (VEGFR), anaplastic lymphoma kinase (ALK), mesenchymalepithelial transition factor (c-MET), v-Raf murine sarcoma viral oncogene homolog B (BRAF), etc (Ai et al., 2018). The occurrence and development of tumours often involve the interaction of multiple receptors and signaling pathways, which makes it difficult to achieve satisfactory effect using single-targeted drugs (Zheng et al., 2017). In recent years, combination drug therapy has gradually become the focus of cancer treatment to improve the efficacy and overcome drug resistance such as the combination use of apatinib with icotinib (Xia et al., 2018), dovitinib with erlotinib (Das et al., 2015) for the treatment of NSCLC. However, the use of polypharmacotherapy makes drug-drug interactions due to inhibition or induction of drug-metabolizing enzymes and/or transporters virtually unavoidable, which may cause serious adverse events and even lead to the early termination of development or withdrawal of drugs from the market, such as terfenadine, astemizole, cisapride, and mibefradil (Alfaro, 2001). .

Ningetinib tosylate (CT-053PTSA, N-(3-fluoro-4-((7-(2-hydroxy-2-methylpropoxy)quinolin-4yl)oxy)phenyl)-1,5-dimethyl-3-oxo-2-phenyl-2,3-dihydro-1H-pyrazole-4-carboxamide,Fig 1A) is a potent, orally bioavailable small molecule tyrosine kinase inhibitor (TKI) against c-MET, VEGFR as well as AXL, Mer, and FMS-like tyrosine kinase 3 (FLT3) in relation to tumour pathogenesis. It's now undergoing phase I/II clinical study for NSCLC treatment (Wang. \& Jin, 2018; Xi, Zhang, Wang, Wu, \& Wang, 2014). In humans, $N$-demethylated ningetinib (M1, Fig 1B) was identified as the primary circulating metabolite, whose plasma exposure was about 1.7-fold that of the parent drug. Although M1 had almost no inhibitory effect on the antitumour targets antagonised by ningetinib, it should cause our concern in clinical studies of ningetinib because of its high plasma exposure.

Gefitinib is the first EGFR-targeting agent launched as an anti-cancer drug in Japan, Australia and the United Sates for the treatment of chemoresistant NSCLC (Rahman, Korashy, \& Kassem, 2014; Ranson \& Wardell, 2004) and was approved for the first-line treatment for metastatic NSCLC and granted orphan drug qualification by FDA (Kazandjian et al., 2016). However, resistance to EGFR-TKIs is inevitable due to various mechanisms, such as the secondary mutation (T790M), activation of alternative pathways (c-MET, HGF, AXL) and aberrance of the downstream pathway (K-RAS mutations, loss of PTEN), etc (Huang \& Fu, 2015). Therefore, it would be reasonable to explore the feasibility and tolerability of combining EGFR-TKIs with multi-target TKIs in the treatment of NSCLC, such as co-administration of gefitinib with ningetinib.

To examine the effect of concomitant medication use on the clinical efficacy and safety, the potential pharmacokinetic interaction of therapeutic doses of gefitinib $(250 \mathrm{mg})$ and ningetinib $(60 \mathrm{mg})$ was firstly evaluated in patients with NSCLC. When co-administered with gefitinib, the plasma exposure of the primary circulating component $\mathrm{M} 1$ on the first and 28th days was reduced by more than $80 \%$, suggestive of a drug-drug interaction between ningetinib and gefitinib. Nevertheless, it is interesting to note that the pharmacokinetics of ningetinib was almost unaffected. Several studies have shown gefitinib inhibited CYP2C19, CYP2D6, CYP2C9, CYP3A4, CYP1A2 and CYP2C8 activities to varying degrees (Filppula, Neuvonen, \& Backman, 2014; Rahman et al., 2014). In addition, gefitinib was also an inhibitor of the efflux transporters 
P-glycoprotein (P-gp) and breast cancer resistance protein (BCRP) (Galetti et al., 2015a; Kitazaki et al., 2005). To date, it was unknown whether inhibition of these metabolizing enzymes and transporters by gefitinib affected the pharmacokinetics of M1. Besides, it was puzzling that the plasma concentration of the parent drug has hardly changed when that of M1 with a high plasma exposure was dramatically reduced.

Hence, the purpose of the present study was to investigate the mechanism of M1 formation and the effect of gefitinib on M1 production, and further explore the reasons for the different effects on ningetinib and M1 by gefitinib using a variety of in vitro and in vivometabolic and transport models.

\section{Materials and Methods}

\subsection{Chemicals and reagents.}

Ningetinib, M1, deuterated ningetinib (D6-ningetinib) and deuterated M1 (D6-M1) were kindly provided by Sunshine Lake Pharma Co., Ltd. (Guangdong, China). Digoxin, imatinib, gefitinib and vincristine were purchased from Dalian Meilun Biotechnology Co., Ltd. (Liaoning, China). Human recombinant CYP450 enzymes (CYP1A1, CYP1A2, CYP2A6, CYP2B6, CYP2C8, CYP2C9, CYP2C19, CYP2D6, CYP2E1, CYP2J2, CYP3A4, CYP3A5, CYP4A11 and CYP4F2) and pooled human intestinal microsomes (HIMs), human kidney microsomes (HKMs) and human lung microsomes (HLUMs) were obtained from BD Gentest (Woburn, MA). Pooled human liver microsomes (HLMs) were supplied by Reid Liver Disease Research Co., Ltd. (Shanghai, China). Primary human hepatocytes were obtained from Sekisui XenoTech (Kansas City, Kansas). Nicotinamide adenine dinucleotide phosphate (NADPH), GF120918, KO143 and sodium carboxymethyl cellulose (CMC-Na) were purchased from Sigma-Aldrich (St. Louis, MO). TAK-875 was obtained from Shanghai Biochempartner Co., Ltd. (Shanghai, China). William's Medium E (WME), DMEM medium, foetal bovine serum (FBS), L-glutamine, non-essential amino acids, $0.25 \%$ trypsin, penicillin G and streptomycin were purchased from Invitrogen (Carlsbad, CA). Deionised water was generated using a Millipore Milli-Q gradient water purification system (Molsheim, France). The Madin-Darby canine kidney (MDCKII)-MDR1 (P-gp transfected cells), MDCKII-BCRP and MDCKII-MRP2 cells were kindly provided by Professor Hongjian Zhang from the School of Pharmacy, Soochow University (Jiangsu, China).

\subsection{Animals.}

The ICR mice (18-20 g in weight, male) were purchased from Sipper-BK Laboratory Animal Co., Ltd. (Shanghai, China). All animal studies were performed in accordance with the principles of the Animal Care and Use of Laboratory Committee of the Shanghai Institute of Materia Medica, Chinese Academy of Sciences (Shanghai, China).

\subsection{Subjects, treatment and sample collection.}

The patients were enrolled at Sun Yat-sen University Cancer Center. According to the Helsinki Declaration, the design and monitoring of clinical studies were consistent with the ethical principles of Good Clinical Practice. The key inclusion and exclusion criteria are listed in Table S1. Each eligible study subject was required to read, sign and date a consent form prior to inclusion in the study. A single oral dose (60 mg) of ningetinib capsules was administered every $24 \mathrm{~h}$ to patients with NSCLC ( $\mathrm{n}=5$, aged 28.5-45.9 years old, body weight of $51.9-73.8 \mathrm{~kg}$, height of $158-188 \mathrm{~cm}$ ) from day 1 to day 28 . In another clinical trial, a single oral dose of ningetinib capsules and $250 \mathrm{mg}$ of gefitinib tablets were daily administered to the patients with NSCLC ( $\mathrm{n}=4$, aged 47-76 years old, body weight of $55.2-68.6 \mathrm{~kg}$, height of 149-170.5 cm) for 28 days. On the first and 28th days, venous blood samples were collected within $1 \mathrm{~h}$ before administration and $0.5,1,2$, $3,4,5,8,12$ and $24 \mathrm{~h}$ in tubes containing EDTA-2K. All the blood samples were centrifuged at $4{ }^{\circ} \mathrm{C}$ at 2000 $\mathrm{rpm}$ for $10 \mathrm{~min}$. The plasma samples were stored at approximately $-70{ }^{\circ} \mathrm{C}$ until analysis.

\subsection{In vitro metabolic stability experiments.}

To investigate the metabolic stability of ningetinib and the formation of M1, primary human hepatocytes were incubated at $37^{\circ} \mathrm{C}$ in $\mathrm{WME}$ at $1.0 \times 10^{6}$ cells $/ \mathrm{mL}$ containing $3 \mu \mathrm{M}$ ningetinib. The HLMs, HIMs, HKMs or HLUMs were incubated at $0.5 \mathrm{mg} / \mathrm{mL}$ in $100 \mathrm{mM}$ phosphate buffer containing $3.2 \mathrm{mM} \mathrm{MgCl}{ }_{2}(\mathrm{PBS}, \mathrm{pH}$ 
7.4) and ningetinib $(3 \mu \mathrm{M})$ in the presence of NADPH $(2 \mathrm{mM})$. The volume of each incubation mixture was $100 \mu \mathrm{L}$. The reactions were terminated by adding equal volume of ice-cold acetonitrile at $0,5,15,30,60$ and $180 \mathrm{~min}$ after the initiation of the reactions. All the incubation samples were triplicated and stored at -20 ${ }^{\circ} \mathrm{C}$ for LC-MS/MS analysis.

\subsection{Enzyme phenotype of M1 production and determination of inhibition kinetics by gefitinib.}

The in vitro metabolism experiments showed that M1 was mainly formed in liver microsomes. Therefore, the enzyme phenotype of M1 formation was evaluated using CYP1A1, CYP1A2, CYP1B1, CYP2A6, CYP2B6, CYP2C8, CYP2C9, CYP2C19, CYP2D6, CYP2E1, CYP2J2, CYP3A4, CYP3A5, CYP4A11 and CYP4F2 in our previous work. The CYP1A1, CYP1B1, CYP2C9 and CYP3A4 could catalyze the metabolism of ningetinib into M1. The gefitinib inhibition on the formation of M1 was explored in CYP1A1, CYP1B1, CYP2C9, CYP3A4 and HLMs incubations. After optimisation of the enzyme concentration and incubation time, the incubation conditions in the linear range were selected. The reaction mixture containing 100 mM PBS (pH 7.4) supplemented with CYPs $\left(5,10,5\right.$ or $10 \mathrm{pmol} \cdot \mathrm{mL}^{-1}$ of CYP1A1, CYP1B1, CYP2C9 or CYP3A4, respectively) or HLMs $\left(0.05 \mathrm{mg} \cdot \operatorname{protein} \cdot \mathrm{mL}^{-1}\right)$ was incubated with $0.01-30 \mu \mathrm{M}$ gefitinib. In addition, different concentrations of ningetinib were added to the CYP1A1 $(0.1,0.3,0.9,2.7$ or $8 \mu \mathrm{M})$, CYP1B1 $(0.05,0.1,0.2,0.5$ or $1 \mu \mathrm{M})$, CYP2C9 $(3,6,12$ or $30 \mu \mathrm{M})$, CYP3A4 $(3,5,15,30$ or $60 \mu \mathrm{M})$ and HLMs reaction mixtures $(1,3,6,10$ or $30 \mu \mathrm{M})$. The reactions were initiated by the addition of NADPH $(2$ $\mathrm{mM}$ ). The volume of each reaction mixture was $100 \mu \mathrm{L}$. An equal volume of ice-cold acetonitrile was added to terminate the reaction at 5, 15,60,30 and $60 \mathrm{~min}$ after the reaction's initiation. All incubation samples were triplicated and stored at $-20{ }^{\circ} \mathrm{C}$ before LC-MS/MS analysis.

\subsection{Tissue distribution of ningetinib and M1 in mice.}

A total of 18 healthy male ICR mice were randomly divided into six groups, which were fasted for $12 \mathrm{~h}$ with free access to water and received a single oral administration of ningetinib at $10 \mathrm{mg} \mathrm{kg}^{-1}$ (suspended in $0.5 \%$ CMC-Na solution; the ningetinib dose used here in mice was basically equivalent to the clinical dosage in NSCLC patients when corrected for interspecies differences according to the Meeh-Rubner formula.). Each group of three mice was sacrificed via exsanguination from the abdominal aorta under anaesthesia at 0,2 , $6,12,24$ or $48 \mathrm{~h}$ after administration. The blood, brain, heart, liver, spleen, lung, kidney, bladder, pancreas, skeletal muscle (hind limb), testis, stomach wall, small intestine, large intestine, abdominal fat, adrenal gland and thymus were rapidly collected. The tissues were washed by saline to rinse out contents or blood, weighed and homogenised in five volumes of acetonitrile-water $(1: 1, \mathrm{v} / \mathrm{v})$ after wiping with filter paper. Plasma was obtained by centrifugation of blood at $11,000 \mathrm{~g}$ for $10 \mathrm{~min}$. All samples were stored at $-20^{\circ} \mathrm{C}$ until LC-MS/MS analysis.

\subsection{Transport stud ies.}

The MDCKII-MDR1/BCRP/MRP2 cells were cultured in DMEM supplemented with 10\% FBS, 2 mM Lglutamine, $100 \mathrm{U}$ penicillin $\mathrm{G}, 100 \mu \mathrm{g} \mathrm{mL^{-1 }}$ streptomycin, and $1 \%$ minimum essential medium nonessential amino acids at $37^{\circ} \mathrm{C}$ in a humidified $5 \% \mathrm{CO}_{2}$ atmosphere. The cells were seeded at a density of $2 \times 10^{5}$ cells $/ \mathrm{cm}^{2}$ on a polycarbonate membrane filter membrane on Transwell inserts (Millipore, Billerica, MA). The medium was routinely replaced with a fresh one every other day. The MDCKII-MDR1/BCRP/MRP2 cell lines were grown for 4 days before the bidirectional transport study.

For substrate assessment, the apical and basolateral chambers were washed twice with prewarmed HBSS $(\mathrm{pH}$ 7.4), and then, the cells were equilibrated for $30 \mathrm{~min}$ in the presence or absence of positive inhibitors

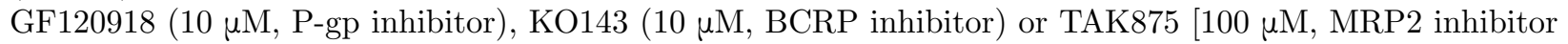
(Li, Zhong, Guo, Zhong, \& Chen, 2015)]. Equilibration HBSS was removed at the end of the equilibrium. HBSS containing ningetinib $(10 \mu \mathrm{M})$ or $\mathrm{M} 1(10 \mu \mathrm{M})$ was added to the donor side (either the apical or basolateral chamber), and the positive inhibitors were added to both chambers. The cells were incubated for $90 \mathrm{~min}$ at $37^{\circ} \mathrm{C}$, at which time aliquots $(150 \mu \mathrm{L})$ were collected from the receiver chambers for analysis. The samples were stored at $-20{ }^{\circ} \mathrm{C}$ for LC-MS/MS analysis. Digoxin (P-gp positive substrate), imatinib (BCRP positive substrate) and vincristine (MRP2 positive substrate) were used for validation of the above efflux 
system.

Given that the metabolite M1 was eliminated more slowly than the parent drug in the plasma, the effects of ningetinib and gefitinib on the efflux of M1 mediated by P-gp, BCRP and MRP2 were further evaluated. Experimental procedures were the same as above, whereas the positive inhibitors were replaced by ningetinib $(0-100 \mu \mathrm{M})$ or gefitinib $(0-100 \mu \mathrm{M})$. All samples were stored at $-20{ }^{\circ} \mathrm{C}$ before LC-MS/MS analysis.

\subsection{Effect of ningetinib on the pharmacokinetics of D6-M1 in mice.}

Fourteen healthy male ICR mice were randomly divided into treatment and control groups. They were fasted for $12 \mathrm{~h}$ with free access to water before the experiments. In the treatment group, ningetinib $\left(40 \mathrm{mg} \cdot \mathrm{kg}^{-1}\right.$, suspended in $0.5 \%$ CMC-Na solution; This dose design considered the high initial blood concentration of D6-M1 when administered intravenously.) was administrated to mice by gavage $30 \mathrm{~min}$ in advance, and then, D6-M1 (0.5 mg. $\mathrm{kg}^{-1}$, dissolved in saline containing 5\% DMSO and 5\% Tween 80) was given through tail vein injection. In the control group, the same volume of $0.5 \% \mathrm{CMC}-\mathrm{Na}$ solution $\left(10 \mathrm{~mL} \cdot \mathrm{kg}^{-1}\right)$ without ningetinib was orally administered. Then, $30 \mathrm{~min}$ later D6-M1 $\left(0.5 \mathrm{mg} \cdot \mathrm{kg}^{-1}\right)$ was injected intravenously. Blood samples ( $5 \mu \mathrm{L}$ for each) were collected through the tail vein and placed in test tubes containing $30 \mu \mathrm{L}$ of $0.1 \mathrm{M}$ trisodium citrate solution before administration $(0 \mathrm{~h})$ and $0.083,0.25,0.5,1,2,4,6,8,12,24$ and $48 \mathrm{~h}$ after administration of D6-M1. Blood samples were stored at $-20{ }^{\circ} \mathrm{C}$ until LC-MS/MS analysis.

\subsection{Instruments and sample preparation.}

Quantitation analysis of ningetinib and M1 was conducted using an API 5500 triple quadrupole mass spectrometer coupled with an LC-30AD high-performance liquid chromatography system (Shimadzu, Kyoto, Japan). Data acquisition and processing was conducted using Analyst 1.6.3 software (AB Sciex, MA, US). Chromatographic separation was achieved on YMC-Triart C18 $(50 \mathrm{~mm} \times 2.0 \mathrm{~mm}$ i.d., $5 \mu \mathrm{m}$; YMC Karasuma-Gojo Bldg, Japan) at $40{ }^{\circ} \mathrm{C}$. The mobile phase was a mixture of $5 \mathrm{mM}$ ammonium acetate (A) and acetonitrile (B) at a flow of $0.6 \mathrm{~mL} \mathrm{~min}{ }^{-1}$. The gradient conditions were as follows: $20 \% \mathrm{~B}$ for $0.5 \mathrm{~min}$; a stepwise linear increase to $80 \% \mathrm{~B}$ at $1.5 \mathrm{~min} ; 1.5-2 \mathrm{~min}, 80 \% \mathrm{~B}$; a stepwise linear decrease to $20 \% \mathrm{~B}$ at $2.5 \mathrm{~min} ; 2.5-3 \mathrm{~min}$, $20 \%$ B. Multiple reaction monitoring (m/z 557.3 - 215.0 for ningetinib, m/z 563.4 - 215.3 for D6-ningetinib, $\mathrm{m} / \mathrm{z} 543.1-271.1$ for M1 and m/z 549.4 - 271.5 for D6-M1) was used in the positive electrospray ionisation mode with an ion spray voltage of $4500 \mathrm{~V}$ and a source temperature of $400 \mathrm{degC}$. The nebuliser gas, heater gas and curtain gas were set to 50,50 and 20 psi, respectively. All the in vitro and in vivo samples were prepared by protein precipitation with acetonitrile.

\section{Data analysis.}

Experimental procedures and statistical analysis were double-blind designed. The data and statistical analyses comply with the recommendations on experimental design and analysis in pharmacology (Curtis et al., 2018).

In the pharmacokinetic study, WinNonlin software (version 6.1; Pharsight Corp., Cary, NC) was used to calculate the pharmacokinetic parameters in a noncompartmental model.

For the inhibition kinetics studies, $\mathrm{V}_{\max }$ and $\mathrm{K}_{\mathrm{m}}$ values were determined by the nonlinear regression curve fit using the Michaelis-Menten equation. Intrinsic clearance $\left(\mathrm{CL}_{\mathrm{int}}\right)$ was calculated as $C \mathrm{~L}_{\mathrm{int}}=\mathrm{V}_{\max } / \mathrm{K}_{\mathrm{m}}$. The apparent kinetic parameters for inhibitory activity $\left(\mathrm{K}_{\mathrm{i}}\right)$ were first estimated by graphical methods such as Dixon (1953) and Cornish-Bowden (1974) methods, and were more accurately determined by nonlinear least square regression analysis on the basis of the best enzyme inhibition model using the Graphpad Prism (version 8.01; GraphPad Software Inc., La Jolla, CA). On the basis of Dixon (1953) and Cornish-Bowden (1974) methods, the linear regression lines obtained in our experiments were all intersected at one point. Thus, the inhibition data were well fitted by the mixed-type inhibition $\mathrm{v}=\left(\mathrm{V}_{\max }[\mathrm{S}]\right) /\left(\mathrm{K}_{\mathrm{m}}\left(1+[\mathrm{I}] / \mathrm{K}_{\mathrm{i}}\right)+\right.$ $[\mathrm{S}]\left(1+[\mathrm{I}] / \alpha \mathrm{K}_{\mathrm{i}}\right)$. The models tested included pure and partial competitive, noncompetitive, uncompetitive and mixed-type inhibitions. 
In the transport experiments, the apparent permeability coefficients $\left(\mathrm{P}_{\mathrm{app}}\right)$ and efflux ratio $(\mathrm{ER})$ was calculated using the following formula:

$\mathrm{P}_{\text {app }}=\mathrm{C}_{\mathrm{T}} \times \mathrm{V} /\left(\mathrm{C}_{0} \times \mathrm{T} \times \mathrm{S}\right)$

$\mathrm{ER}=\mathrm{P}_{\text {app, }} \mathrm{B}$ to A $/ \mathrm{P}_{\text {app }}$, A to B

where $\mathrm{C}_{\mathrm{T}}=$ the concentration of the test compound on the receiver side, $\mathrm{V}=$ the loading volume on the receiver side, $\mathrm{S}=$ the surface area of the cell monolayer $\left(0.33 \mathrm{~cm}^{2}\right.$ in a 24 -well plate $), \mathrm{C}_{0}=$ the initial concentration of the test compound on the donor side and $\mathrm{T}=$ incubation time. $\mathrm{P}_{\mathrm{app}}, \mathrm{A}$ to $\mathrm{B}$ and $\mathrm{P}_{\mathrm{app}}, \mathrm{B}$ to $\mathrm{A}$ represent the extent of permeation generated by the transport from the apical to basolateral sides and from the basolateral to apical sides, respectively.

In the transport inhibition study, the $\mathrm{IC}_{50}$ values were calculated by plotting the log value of the inhibitor concentration against the normalised response as follows: $\mathrm{Y}=100 /\left[1+10^{\text {(X-Log(IC50) }}\right]$.

Statistical comparisons between two groups were evaluated using the unpaired student's t-test in GraphPad Prism. $P<0.05$ was considered significant.

\section{Results}

\subsection{Pharmacokinetic interaction of ningetinib with gefitinib in patients with NSCLC.}

As shown in Fig 2 and Table 1, when ningetinib was given alone, the peak concentrations $\left(C_{\max }\right)$ of ningetinib and $\mathrm{M} 1$ in plasma were comparable, and $\mathrm{AUC}_{0-24 \mathrm{~h}}$ value of $\mathrm{M} 1$ was approximately 1.7-fold that of ningetinib. Moreover, the time to reach the peak concentration $\left(\mathrm{T}_{\max }\right)$ of $\mathrm{M} 1$ was significantly longer than that of ningetinib, and the elimination rate of M1 was much slower than that of ningetinib. After co-administration with gefitinib, the $\mathrm{C}_{\max }$ and $\mathrm{AUC}_{0-24 \mathrm{~h}}$ of ningetinib were almost unchanged, whereas those values for M1 significantly dropped by more than $80 \%$ on the first and 28th day. The elimination behaviours of both ningetinib and M1 were not obviously changed. These data suggested a DDI between ningetinib and gefitinib, mostly likely through metabolic mechanism.

\subsection{Investigation of M1 formation in vitro.}

The mechanism of M1 formation was initially explored by in vitrometabolic incubation system. After incubation for 180 min, less than $10 \%$ of ningetinib was consumed, mainly in the liver microsomes (Fig 3). A small amount of M1 $(<5 \%)$ was detected in HLMs and even less in HIMs. M1 was not detected in HKMs and HLUMs.

\subsection{P450 enzymes responsible for M1 production and inhibition kinetics by gefitinib.}

Previous study in our laboratory showed that among the 15 kinds of P450 enzymes examined, CYP1A1, CYP1B1, CYP2C9 and CYP3A4 could catalyze the metabolism of ningetinib into M1. According to the $\mathrm{CL}_{\text {int }}$ values shown in Table 2, CYP1A1 played a predominant role in M1 formation, whereas CYP1B1, CYP2C9 and CYP3A4 had a minor catalytic effect. The inhibition kinetics of gefitinib on HLMs and the four P450 enzymes was further studied. As shown in Fig. 4, gefitinib demonstrated an inhibitive effect on M1 formation in HLMs, CYP1A1, CYP1B1 and CYP2C9 incubations. The inhibition type was determined to be a mixed-type competitive inhibitory nature with $\mathrm{K}_{\mathrm{i}}$ of $16.4,0.095,1.83$ and $9.23 \mu \mathrm{M}$. However, the production of M1 in the CYP3A4 incubation was not inhibited by gefitinib.

\subsection{Tissue distribution of ningetinib and M1 in mice.}

In vitro experiments showed that M1 formation was less than $5 \%$ in HLMs. In vivo experimental evidence that M1 in excreta accounted for $<2 \%$ of the dose administered further supported that $N$-demethylation was not the primary metabolic pathway of ningetinib in humans. To provide a plausible explanation for high plasma exposure of M1, the tissue distribution of ningetinib and M1 was determined in mice because the metabolite profile of ningetinib in mice was similar to that of the NSCLC patients (Table S2 and Figure S1). According to Fig. 5A, after administering $10 \mathrm{mg} \cdot \mathrm{kg}^{-1}$ of ningetinib orally to the mice, the parent drug 
ningetinib exhibited an extensive tissue distribution with a higher exposure in most tissues than in plasma. Unlike the parent drug, the M1 concentrations in tissues other than the liver were all lower than that in plasma (Fig. 5B). Similar to human pharmacokinetics, the plasma concentration of M1 in mice gradually increased over time and was even more than double the parent drug. However, the M1 concentrations in tissues apart from the liver were less than $30 \%$ of the parent drug (Fig. 5C). Plasma was the preferred site for M1 disposition in vivo.

\subsection{Effects of ningetinib and gefitinib on M1 mediated by efflux transporters.}

M1 exhibited a longer plasma elimination half-life than the parent drug in ICR mice and in NSCLC patients, possibly because the efflux pathway of M1 was likely to be blocked. The influence of efflux transporters on the disposition of ningetinib and M1 was further studied.

To determine whether ningetinib or M1 was a substrate of efflux transporters, the MDCKII cells stableoverexpressed with human P-gp (MDCKII-MDR1), BCRP (MDCKII-BCRP) or MRP2 (MDCKII-MRP2) were used for substrate screening (Table 3). The ER values of ningetinib/M1 were all greater than 2 apart from that of ningetinib in MDCKII-MRP2 cells. Then the addition of GF120918 (P-gp inhibitor), KO143 (BCRP inhibitor) and TAK875 (MRP2 inhibitor) markedly reduced the ER values of M1 in MDCKII-MDR1, MDCKII-BCRP and MDCKII-MRP2 cells, respectively, while ningetinib displayed a similar result except for that in MDCKII-MRP2 cells. Thus, both ningetinib and M1 were substrates of P-gp and BCRP. MRP2 mediated the efflux of M1 but not of ningetinib.

The inhibitory effect of ningetinib and gefitinib on P-gp, BCRP and MRP2-mediated M1 efflux was further investigated. The logarithm concentration of ningetinib or gefitinib was plotted on the abscissa, and the remaining activity of P-gp, BCRP or MRP2 (percentage of efflux ratio in the inhibitor group to that in the control group) was plotted on the ordinate, as shown in Fig. 6. Ningetinib displayed a concentrationdependent inhibition on P-gp-, BCRP- and MRP2-mediated M1 efflux with $\mathrm{IC}_{50}$ values of 0.413 (0-100 $\mu \mathrm{M}), 18.7 \mu \mathrm{M}(0-100 \mu \mathrm{M})$ and $3.06 \mu \mathrm{M}(0-30.0 \mu \mathrm{M})$, respectively. Similarly, gefitinib exhibited a weak concentration-dependent inhibition on P-gp- and BCRP-mediated M1 efflux with $\mathrm{IC}_{50}$ of $5.40(0-100 \mu \mathrm{M})$ and $9.09 \mu \mathrm{M}(0-30.0 \mu \mathrm{M})$, respectively, but showed no inhibition on MRP2-mediated M1 efflux (0-100 $\mu \mathrm{M})$. These results, especially the P-gp inhibition phenomenon, indicated that the parent drug ningetinib could potently inhibit the canalicular efflux of its metabolite M1 and thereby elevate its plasma exposure. The inhibitory effect of gefitinib on M1 efflux was significantly weaker than that of ningetinib.

\subsection{Effect of ningetinib on pharmacokinetics of D6-M1 in mice.}

To investigate whether ningetinib could affect the pharmacokinetics of M1 by inhibiting efflux transporters, D6-M1 was intravenously injected to ICR mice to prevent the effect of M1 generated by ningetinib metabolism. The results are shown in Fig. 7 and Table 4. Compared with the control group, the blood exposure of D6-M1 in the ningetinib-combination group was increased by approximately $75 \%$. In addition, the blood concentration of D6-M1 was not affected by ningetinib within $2 \mathrm{~h}$ after administration. From 4 $\mathrm{h}$ to $24 \mathrm{~h}$, the blood exposure of D6-M1 in the ningetinib group increased nearly by $200 \%$, which further demonstrated that ningetinib could inhibit the elimination of D6-M1.

\section{Discussion}

In pharmacokinetic studies, M1 was identified as the primary circulating metabolite of ningetinib in humans. However, in clinical trials on multi-target-based combination medication for NSCLC therapy, both the plasma exposure and $\mathrm{C}_{\max }$ of $\mathrm{M} 1$ dropped dramatically and were reduced by more than $80 \%$ when ningetinib was coadministered with gefitinib, whereas the pharmacokinetic parameters (AUC, $\mathrm{C}_{\max }$ and $\mathrm{T}_{\max }$ ) of ningetinib were not affected. The elimination behaviours of both ningetinib and M1 were not obviously changed. These data suggested a DDI between ningetinib and gefitinib, mostly likely through metabolic mechanism.

The mechanism of M1 formation was initially investigated. The enzymatic kinetic data showed that M1 formation was mainly mediated by CYP1A1 and to a lesser extent by CYP1B1, CYP2C9 and CYP3A4. When examining the potency of gefitinib as an inhibitor of CYP450s in vitro, our results were somewhat 
different from those reported in the literature (Filppula, Neuvonen, \& Backman, 2014; Rahman et al., 2014). The present study revealed that gefitinib was a strong inhibitor of CYP1A1, moderate inhibitor of CYP1B1 and weak inhibitor of CYP2C9 but had no influence on CYP3A4. Gefitinib significantly inhibited M1 production in HLMs, but the concentration of the parent drug was not influenced by gefitinib, since the amount of M1 produced in HLMs and in patients was less than 5\%, and $N$-desmethylation was not a major metabolic pathway of ningetinib in humans.

The high plasma exposure of M1 could be related to tissue distribution and/or clearance. Unlike in plasma, the concentrations of M1 in tissues were less than 30\% of the parent drug, suggesting that the metabolite tended to be retained in the plasma and its penetration into tissues was more difficult than that of ningetinib. One possible explanation for the findings was that M1 exhibited much higher protein binding rates both in mouse and human plasma than the parent drug (99.9\% versus $90 \%)$.

In vitro incubations showed that the liver was the primary site of M1 formation. Thus, the hepatic clearance pathway of M1 was evaluated subsequently. The transport studies demonstrated that M1 was a substrate of P-gp, BCRP and MRP2. The parent drug ningetinib happened to be a potent inhibitor of P-gp and moderate inhibitor of BCRP and MRP2. Therefore, it was speculated that ningetinib obstructed the biliary excretion pathway of M1 and resulted in its high plasma exposure. The D6-M1 pharmacokinetic experiment further proved this conclusion. In addition, the literature (Galetti et al., 2015b; Kitazaki et al., 2005) and our results confirmed that gefitinib was also an inhibitor of P-gp and BCRP. It might inhibit the efflux of M1 mediated by P-gp and BCRP. However, the $\mathrm{IC}_{50}$ value of gefitinib on P-gp was significantly higher than that of ningetinib $(5.40 \mu \mathrm{M}$ versus $0.413 \mu \mathrm{M})$ and gefitinib had no inhibitory effect on MRP2. Though the $\mathrm{IC}_{50}$ value of gefitinib on BCRP was lower than that of ningetinib $(9.09 \mu \mathrm{M}$ versus $18.7 \mu \mathrm{M})$, both of them were much higher than the peak plasma concentrations of ningetinib $\left(1.37 \mu \mathrm{g} \cdot \mathrm{mL}^{-1}\right)$ and gefitinib $\left(272 \mathrm{ng} \cdot \mathrm{mL}^{-1}\right)$ when therapeutic doses of these drugs were administered to NSCLC patients. The plasma $\mathrm{C}_{\max }$ of gefitinib was less than one third of ningetinib. In this situation, it was speculated that the efflux pathway of M1 was inhibited by ningetinib, whereas the inhibition of efflux by gefitinib was negligible due to its higher $\mathrm{IC}_{50}$ value and much lower in vivo concentrations. When co-administrated with gefitinib, the pharmacokinetic alteration of M1 showed the inhibitory effect of gefitinib on M1 formation, not on its efflux transports. The interaction of ningetinib and gefitinib mediated by P-gp and BCRP was also studied in the present work. The ER values of ningetinib were decreased by not more than $50 \%$ under the effect of $50 \mu \mathrm{M}$ of gefitinib (Table S3). Therefore, transporter-mediated effect of gefitinib on ningetinib could be ignored.

To our knowledge, it was the first time to report that gefitinib was a strong inhibitor of CYP1A1. CYP1A1 was the most actively studied human pulmonary CYP enzyme involved in polycyclic aromatic hydrocarbons metabolism, and might play a role in the development of lung cancer (Oyama et al., 2008). It was also reported that CYP1A1 was highly expressed in human brain, colon and bladder tumour tissues compared to the normal tissues, as determined by mRNA level expression (Androutsopoulos et al., 2013; Wahid, Mahjabeen, Baig, \& Kayani, 2013). In addition, CYP1A1 polymorphisms (rs4646903 and rs1048943) were positively correlated with prostate cancer, breast cancer, lung cancer, et,al (Lu et al., 2020). Therefore, gefitinib's strong inhibitory effect on CYP1A1 may provide possibilities for its application in tumour prevention and reversal of terminal tumour resistance. In vitroexperiments in our laboratory indicated that the amount of M1 produced was positively correlated with the protein expression of CYP1A1 in HLMs and HLUMs from smokers of varying degrees. The high selectivity of ningetinib for CYP1A1 may provide its potential as an indicator of CYP1A1.

In conclusion, this study illustrated the DDI mechanism of ningetinib and gefitinib in patients with NSCLC. Ningetinib was metabolized by CYP1A1 to produce a small amount of $N$-demethylated metabolite M1. The low tissue affinity of M1 and the inhibitory effect of ningetinib on M1 canalicular efflux resulted in its high plasma exposure and long elimination half-life. When co-administrated, as a strong inhibitor of CYP1A1, gefitinib inhibited the formation of M1 and reduced its plasma exposure by more than $80 \%$. However, the pharmacokinetics of the parent drug ningetinib was not influenced because of the low metabolic yield of M1. Due to the relatively high expression of CYP1A1 in pulmonary tumour tissues, the concentration of the 
parent drug ningetinib in the target tissue may be increased by gefitinib. As a consequence, the long-term safety profile and efficacy of ningetinib combined with gefitinib should be concerned in NSCLC patients. The findings also suggested that in-depth analysis of the pharmacokinetics of metabolites in DDI studies can help enrich the DDI mechanism and discovery of novel inhibitors (or inducers) of metabolic enzymes or transporters.

\section{Reference}

Ai, X., Guo, X., Wang, J., Stancu, A. L., Joslin, P. M. N., Zhang, D., \& Zhu, S. (2018). Targeted therapies for advanced non-small cell lung cancer. Oncotarget, 9 (101), 37589-37607. doi:10.18632/oncotarget.26428

Alfaro, C. L. (2001). Emerging role of drug interaction studies in drug development: the good, the bad, and the unknown. Psychopharmacol Bull, 35 (4), 80-93.

Androutsopoulos, V. P., Spyrou, I., Ploumidis, A., Papalampros, A. E., Kyriakakis, M., Delakas, D., . . . Tsatsakis, A. M. (2013). Expression profile of CYP1A1 and CYP1B1 enzymes in colon and bladder tumors. PLoS One, 8 (12), e82487. doi:10.1371/journal.pone.0082487

Curtis, M. J., Alexander, S., Cirino, G., Docherty, J. R., George, C. H., Giembycz, M. A., . . . Ahluwalia, A. (2018). Experimental design and analysis and their reporting II: updated and simplified guidance for authors and peer reviewers. Br J Pharmacol, 175 (7), 987-993. doi:10.1111/bph.14153

Das, M., Padda, S. K., Frymoyer, A., Zhou, L., Riess, J. W., Neal, J. W., \& Wakelee, H. A. (2015). Dovitinib and erlotinib in patients with metastatic non-small cell lung cancer: A drug-drug interaction. Lung Cancer, 89 (3), 280-286. doi:10.1016/j.lungcan.2015.06.011

Filppula, A. M., Neuvonen, P. J., \& Backman, J. T. (2014). In vitro assessment of time-dependent inhibitory effects on CYP2C8 and CYP3A activity by fourteen protein kinase inhibitors. Drug Metab Dispos, 42 (7), 1202-1209. doi:10.1124/dmd.114.057695

Galetti, M., Petronini, P. G., Fumarola, C., Cretella, D., La Monica, S., Bonelli, M., . . . Alfieri, R. R. (2015a). Effect of ABCG2/BCRP Expression on Efflux and Uptake of Gefitinib in NSCLC Cell Lines. PLoS One, 10 (11), e0141795. doi:10.1371/journal.pone.0141795

Galetti, M., Petronini, P. G., Fumarola, C., Cretella, D., La Monica, S., Bonelli, M., . . . Alfieri, R. R. (2015b). Effect of ABCG2/BCRP Expression on Efflux and Uptake of Gefitinib in NSCLC Cell Lines. Plos One, 10 (11).

Huang, L., \& Fu, L. (2015). Mechanisms of resistance to EGFR tyrosine kinase inhibitors. Acta Pharm Sin B, 5 (5), 390-401. doi:10.1016/j.apsb.2015.07.001

Kazandjian, D., Blumenthal, G. M., Yuan, W., He, K., Keegan, P., \& Pazdur, R. (2016). FDA Approval of Gefitinib for the Treatment of Patients with Metastatic EGFR Mutation-Positive Non-Small Cell Lung Cancer. Clinical Cancer Research, 22 (6), 1307-1312.

Kitazaki, T., Oka, M., Nakamura, Y., Tsurutani, J., Doi, S., Yasunaga, M., . . . Kohno, S. (2005). Gefitinib, an EGFR tyrosine kinase inhibitor, directly inhibits the function of P-glycoprotein in multidrug resistant cancer cells. Lung Cancer, 49 (3), 337-343. doi:10.1016/j.lungcan.2005.03.035

Li, X., Zhong, K., Guo, Z., Zhong, D., \& Chen, X. (2015). Fasiglifam (TAK-875) Inhibits Hepatobiliary Transporters: A Possible Factor Contributing to Fasiglifam-Induced Liver Injury. Drug Metab Dispos, 43 (11), 1751-1759. doi:10.1124/dmd.115.064121

Lu, J., Shang, X., Zhong, W., Xu, Y., Shi, R., \& Wang, X. (2020). New insights of CYP1A in endogenous metabolism: a focus on single nucleotide polymorphisms and diseases.Acta Pharm Sin B, 10 (1), 91-104. doi:10.1016/j.apsb.2019.11.016

Malapelle, U., Muscarella, L. A., Pisapia, P., \& Rossi, A. (2020). Targeting emerging molecular alterations in the treatment of non-small cell lung cancer: current challenges and the way forward. Expert Opin Investig 
Drugs , 1-10. doi:10.1080/13543784.2020.1732922

Oyama, T., Sugio, K., Isse, T., Matsumoto, A., Nose, N., Uramoto, H., . . . Kawamoto, T. (2008). Expression of cytochrome P450 in non-small cell lung cancer. Front Biosci, 13 , 5787-5793.

Rahman, A. F., Korashy, H. M., \& Kassem, M. G. (2014). Gefitinib. Profiles Drug Subst Excip Relat Methodol, 39 , 239-264. doi:10.1016/B978-0-12-800173-8.00005-2

Ranson, M., \& Wardell, S. (2004). Gefitinib, a novel, orally administered agent for the treatment of cancer. J Clin Pharm Ther, 29 (2), 95-103. doi:10.1111/j.1365-2710.2004.00543.x

Wahid, M., Mahjabeen, I., Baig, R. M., \& Kayani, M. A. (2013). Expression of CYP1A1 and GSTP1 in human brain tumor tissues in Pakistan. Asian Pac J Cancer Prev, 14 (12), 7187-7191. doi:10.7314/apjcp.2013.14.12.7187

Wang., Y., \& Jin, R. (2018). The research progress of c-Met inhibitors in clinical trials. Cancer Cell Research, 20 , 524-528.

Xi, N., Zhang, Y. J., Wang, Z. H., Wu, Y. J., \& Wang, T. J. (2014). CT053PTSA, a novel c-MET and VEGFR2 inhibitor, potently suppresses angiogenesis and tumor growth.Cancer Research, 74 (19). doi:10.1158/1538-7445.Am2014-1755

Xia, P., Cao, J., Lv, X., Wang, L., Lv, W., \& Hu, J. (2018). Combination therapy of apatinib with icotinib for primary acquired icotinib resistance in patients with advanced pulmonary adenocarcinoma with EGFR mutation. Thorac Cancer, 9 (5), 656-661. doi:10.1111/1759-7714.12624

Zheng, W., Zhao, Y., Luo, Q., Zhang, Y., Wu, K., \& Wang, F. Y. (2017). Multi-Targeted Anticancer Agents. Current Topics in Medicinal Chemistry, 17 (28), 3084-3098.

\section{Legends for Figures}

Fig. 1 Structure of ningetinib (A) and M1 (B)

Fig. 2 Mean plasma concentration-time profiles of ningetinib and M1 in patients with NSCLC following oral administration of $60 \mathrm{mg}$ of ningetinib without (A1/A2) and with (B1/B2) $250 \mathrm{mg}$ of gefitinib.

Fig. 3 Metabolic stability of ningetinib and formation of M1 in human primary hepatocytes (A), human liver microsomes (B), human intestinal microsomes (C), human lung microsomes (D) and human kidney microsomes $(\mathrm{E})$ versus incubation time. Each point represents the mean of three independent experiments $\pm \mathrm{SD}(\mathrm{n}=3)$.

Fig. 4 Inhibitory effect of gefitinib on M1 formation mediated by human recombinant HLMs (A1/A2/A3), CYP1A1 (B1/B2/B3), CYP1B1 (C1/C2/C3) or CYP2C9 (D1/D2/D3). The figures above represent the Dixon (1953) method. The figures in the middle represent the Cornish-Bowden (1974) method. The figures below represent the mixed-type inhibition. Each point represents the mean of three independent experiments $\pm \operatorname{SEM}(\mathrm{n}=3)$. "I" means gefitinib and "S" means ningetinib.

Fig. 5 Tissue distribution of ningetinib and M1 in ICR mice after an oral dose of ningetinib $\left(10 \mathrm{mg} \cdot \mathrm{kg}^{-1}\right)$. (A) ningetinib, (B) M1, (C) M1 to ningetinib ratio. Each point represents the mean of three independent experiments $\pm \mathrm{SD}(\mathrm{n}=3)$.

Fig. 6 Inhibitory effect of ningetinib and gefitinib on M1 efflux in MDCKII-MDR1 (A1/A2), MDCK IIBCRP (B1/B2) and MDCK II-MRP2 (C1/C2) cells. Each point represents the mean of three independent experiments. Remaining activity means percentage of ER values of M1 in the inhibitor group versus the control group.

Fig. 7 Mean blood concentration-time profiles of D6-M1 in mice following intravenous injection of 0.5 $\mathrm{mg} / \mathrm{kg}$ D6-M1 with or without $40 \mathrm{mg} \cdot \mathrm{kg}^{-1}$ ningetinib. Each point represents the mean of three independent experiments $\pm \mathrm{SD}(\mathrm{n}=7) .{ }^{*} \mathrm{p}<0.05,{ }^{* *} \mathrm{p}<0.01,{ }^{* * *} \mathrm{p}<0.001$ vs. the control 


\section{Hosted file}

Table 1.docx available at https://authorea.com/users/303604/articles/433788-drug-interactionof-ningetinib-and-gefitinib-involving-cyp1a1-and-efflux-transporters-in-non-small-celllung-cancer-patients

\section{Hosted file}

Table 2.docx available at https://authorea.com/users/303604/articles/433788-drug-interactionof-ningetinib-and-gefitinib-involving-cyp1a1-and-efflux-transporters-in-non-small-celllung-cancer-patients

\section{Hosted file}

Table 3.docx available at https://authorea.com/users/303604/articles/433788-drug-interactionof-ningetinib-and-gefitinib-involving-cyp1a1-and-efflux-transporters-in-non-small-celllung-cancer-patients

\section{Hosted file}

Table 4.docx available at https://authorea.com/users/303604/articles/433788-drug-interactionof-ningetinib-and-gefitinib-involving-cyp1a1-and-efflux-transporters-in-non-small-celllung-cancer-patients
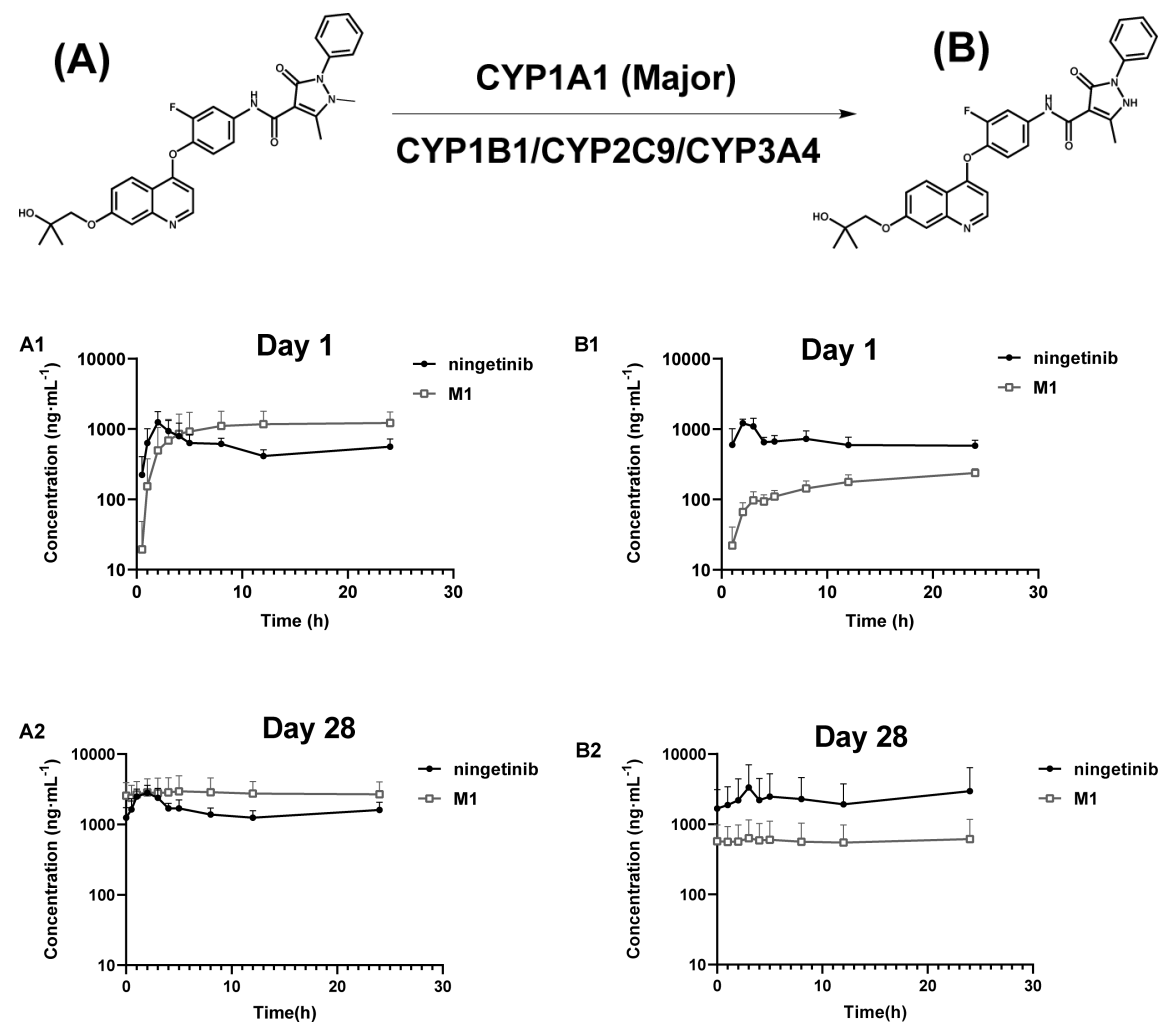
A

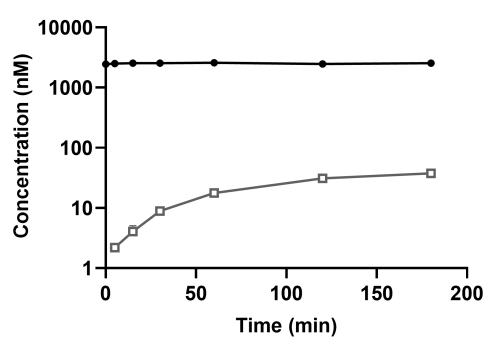

C

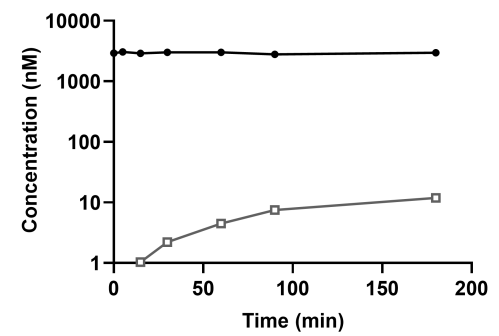

E

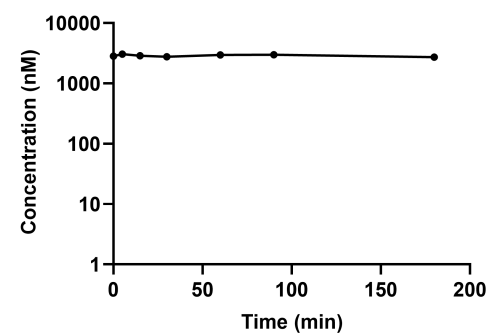

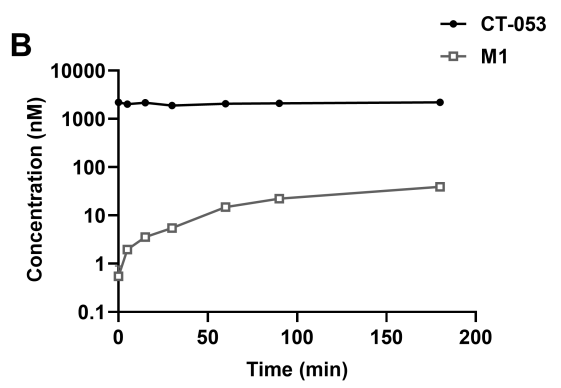

D

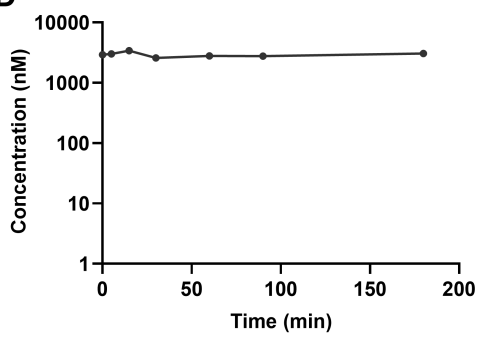

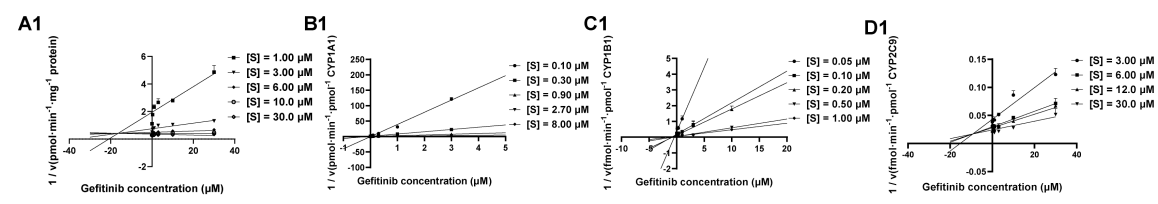
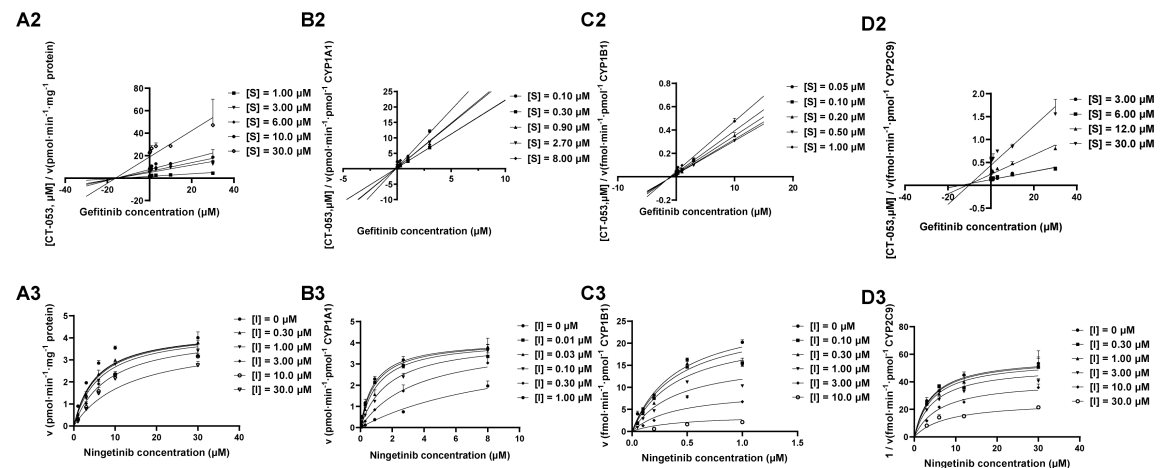


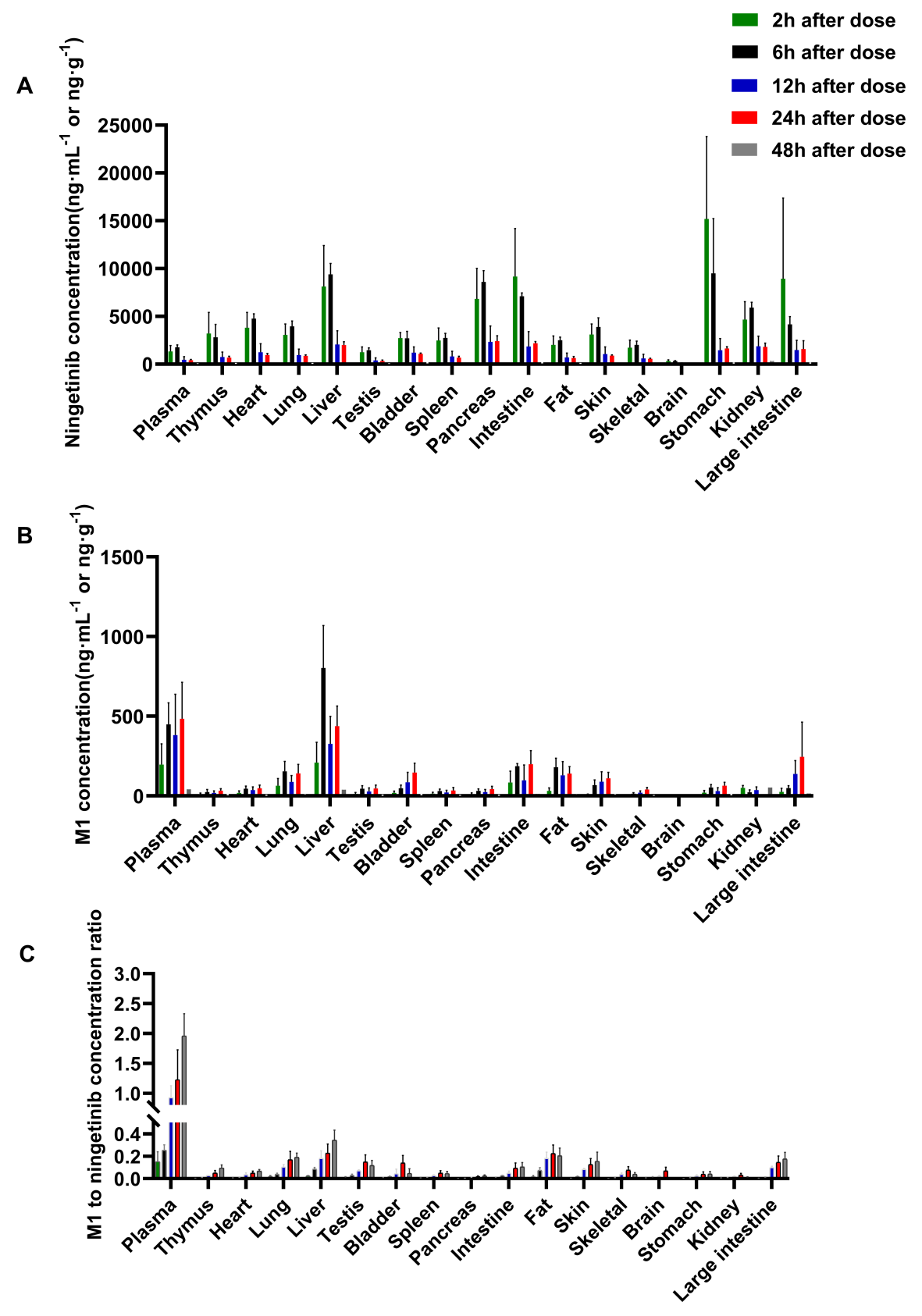



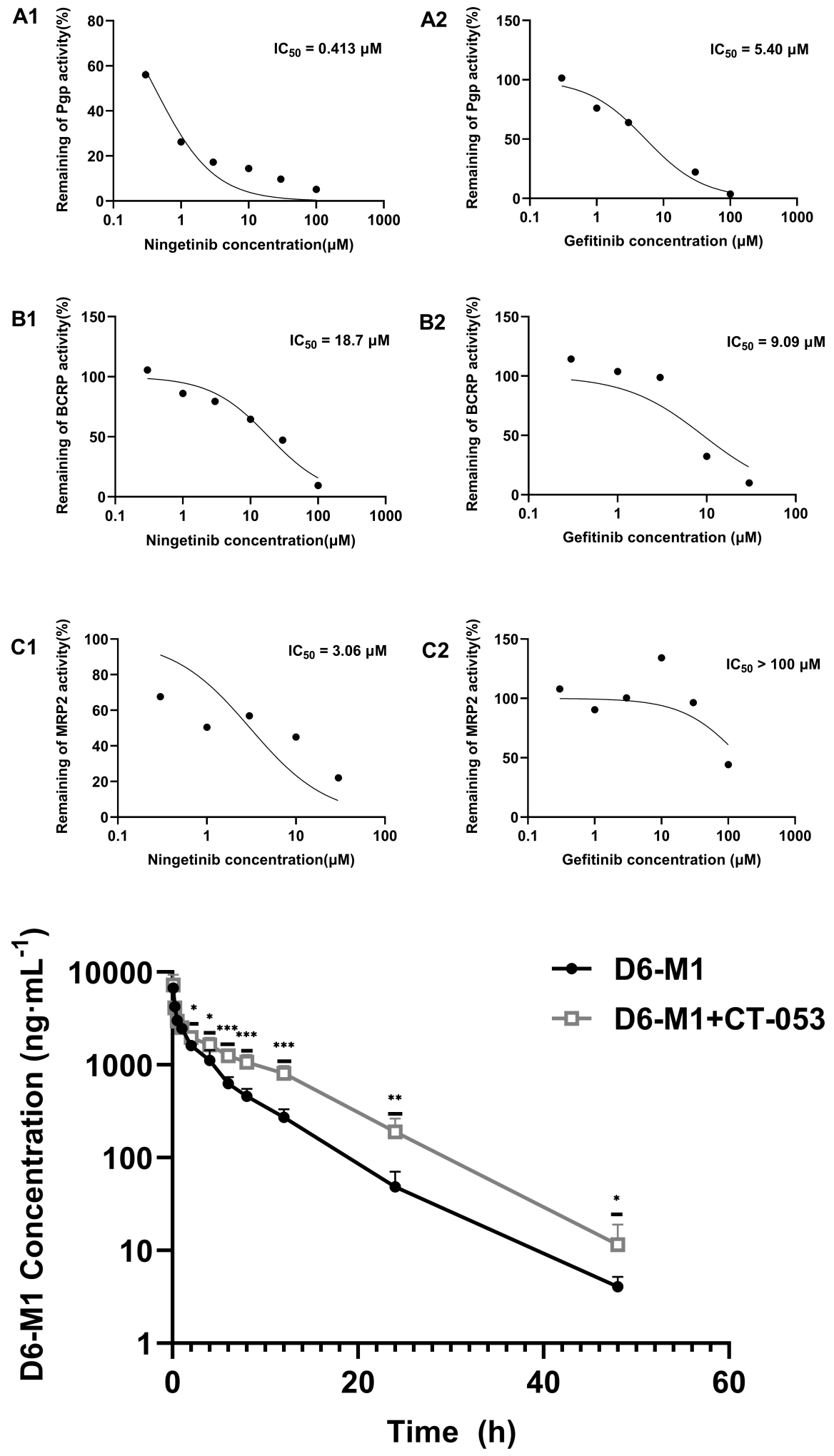